\title{
Take a break
}

Many people have been unable to travel due to the pandemic, or even take a 'staycation'. This August, we encourage you to slow down, look after your mental health, and let these stunning photographs transport you to a different place.

ach year, the Royal Observatory

Greenwich hosts the prestigious Astronomy Photographer of the Year competition. Now in its 13 th year, the entries - more than 4,500 of them taken across 75 countries - are as awe-inspiring as ever. What better way to review our place in the world? Even if that place is a frozen world, as shown in the panoramic image of the Aurora Borealis over Vik, Iceland, in winter. To view the 2021 shortlist, please click here. Winners will be announced on 16 September.
We wish you happy travels, even if you do remain in your chair.

Published online: 13 August 2021

https://doi.org/10.1038/s41550-021-01466-1

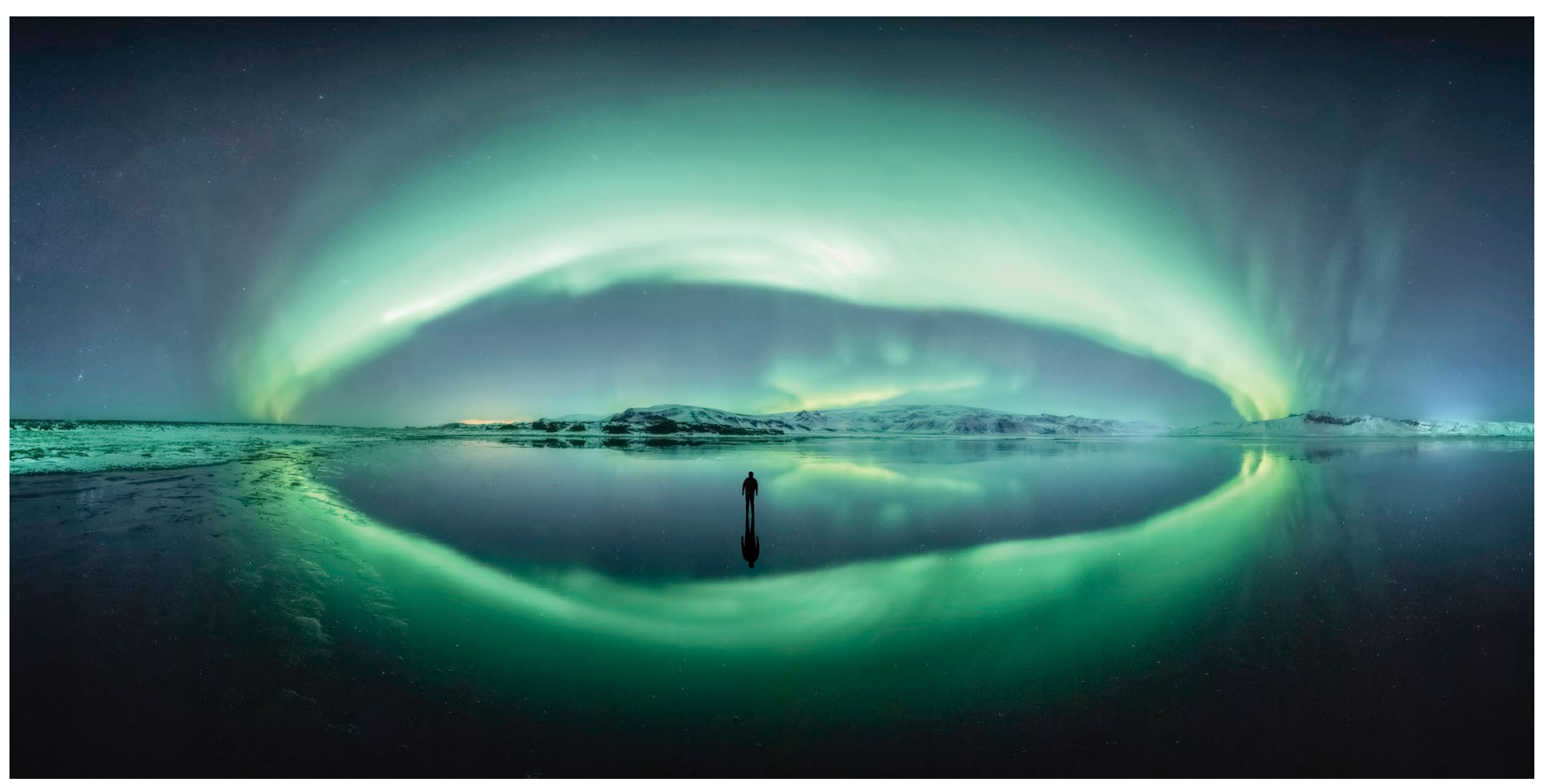

Iceland Vortex. Credit: Larryn Rae 